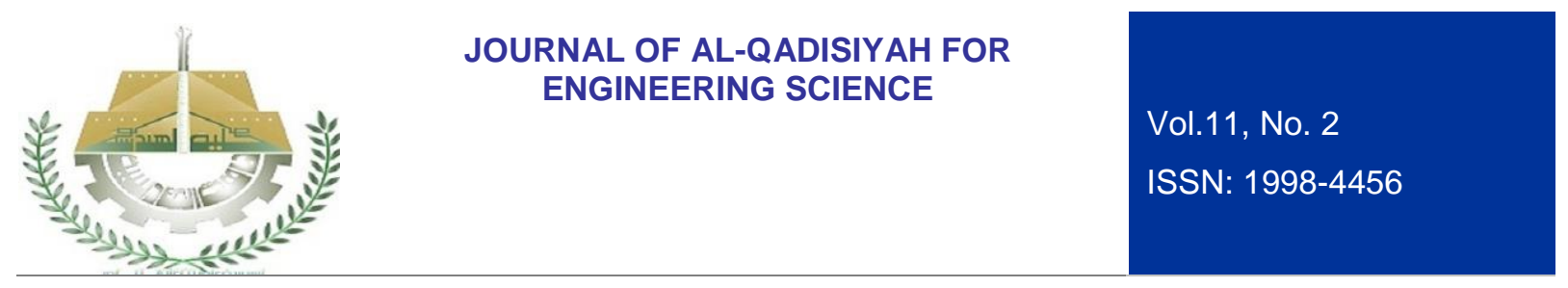

\title{
PERFORMANCE EVALUATION OF SINGLE CARRIER FREQUENCY DOMAIN EQUILIZER (SC-FDE) OVER IMPULSIVE NOISE CHANNEL
}

\author{
Hussein Abdullah Leftah, \\ h.a.leftah@stu.edu.iq \\ Southern Technical University, Basrah Technical Engineering College, Electrical Engineering, Basrah, Iraq. \\ Husham Lateef Swadi, \\ hisham.romi@uobasrah.edu.iq \\ Basrah University, College of Engineering, Electrical Engineering , Basrah, Iraq.
}

\begin{abstract}
Impulsive noise is considered as one of the major source of disturbance in the state-of-the-art multicarrier (MC) communication systems. Therefore, several techniques are being constantly proposed to eliminate the effect of such noise. In this work, a time domain matrix interleaved is compiled with a single carrier frequency domain equalizer (SC-FDE) is proposed to reduce the deleterious effects of impulsive noise. A mathematical model for the proposed scheme is also presented in this paper. Simulation results show that the proposed technique superiors the interleaved multicarrier system where the proposed scheme can completely avoid the error floors not only at high signal-to-noise ratio (SNR) but also at heavily distributed impulsive noise. The bit-error-rate (BER) of the alternative proposed scheme decreases as the signal-to-noise ratio (SNR) increases whereas the BER of the standard system suffers from error-floors with a constant BER at about $10^{-3}$ for about $8 \mathrm{~dB}$ SNR for medium and heavily impulsive noise.
\end{abstract}

Keywords: Multicarrier (MC), Single carrier- Frequency Domain Equalization (SC-FDE), Impulsive Noise (IN), channel modelling, Interleave.

\section{INTRODUCTION}

Single carrier-frequency domain equalizer (SC-FDE) is a technique that has a noticeable attention among other wireless modulation schemes in the last ten years. The SC-FDE system has compiled the popular attributes of both single carrier systems and multicarrier systems. As a single carrier, the SC-FDE has low Peak-to-average power ratio (PAPR), not sensitive to amplifier nonlinearity, tolerance to carrier frequency offset sensitivity. On the other hand, in comparison to multicarrier modulation, the SC-FDE system is robust to multipath fading channels, efficient to intersymbol interference (ISI) mitigation [1] and it has simple equalization. Thus it has been taken up in several wireless applications such as Long-Term evolution (LTE), ultra-wide band (UWB), IEEE 802.11 and HIPERLAN/2.

In the higher frequencies, additional attention will be recruited and highly frequency selective called impulsive noise, hence that the transmitted data may be damaged by impulsive noise in addition to the other noise like the colored Gaussian noise and narrowband interference [2]. Modelling of Electromagnetic interference (EMI) can be separated into two approaches, physical modelling and statistical-physical modelling. The statisticalphysical examples are chosen because they are more accurate models than the physical models due to the mix between natural and human-made sources [3]. Noise generally limited the channel capacity where the system performance be downgraded. One of the most important character of noise is that affected by the new modulation schemes is impulsive noise. Impulsive noise can be defined as relatively short duration "on/off" switching noise pulses, generated from a different of sources the sources of impulsive noise are generated for 


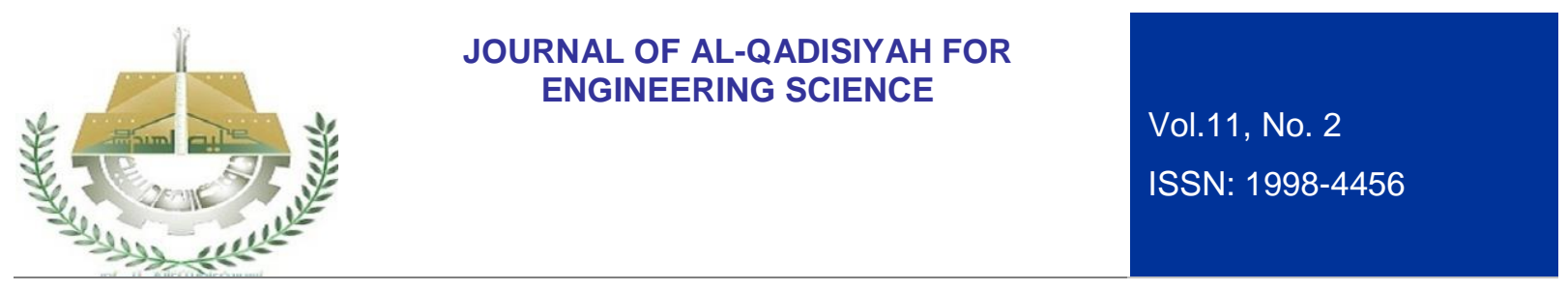

all cases of trading operations in electrical devices [4]. The other source of impulsive noise came from the modelling of interferences between the adjacent channels in mobile communication [5]. Asynchronous impulsive noise levels can reach $50 \mathrm{~dB}$ more than thermal noise so that this value causes a degradation in communication system performance [6,7]. Thus, the communication system should be designed carefully to cope with the impulsive noise.

This paper, proposes SC-FDE modulation technique for applications over impulsive noise channel such as digital audio broadcasting (DAB), digital video broadcasting (DVB) and power line communications (PLC). Also suggests an efficient interleaving technique to further reduction in the effects of the impulsive noise. To clarify the contribution of this theme, it is listed in four points equally

- Propose the SC-FDE modulation for applications with impulsive noise effects instead of the multicarrier digital modulation systems.

- Presents an analytical, mathematical model for the proposed scheme in the presence of impulsive noise.

- Matrix-Interleaved time domain SC-FDE is also investigated in this paper as a solution to severe impulsive noise channel applications.

- Monte Carlo computer simulations are implemented to establish the efficacy of the proposed SC-FDE technique over the conventional multicarrier modulation technique.

\section{IMPULSIVE NOISE MODEL AND EFFECT}

A definition is required to identify an impulsive noise from colored (background) noise, when measuring impulse noise.There is a certain level (Threshold) is identified, and any variation exceeds this threshold level is called impulsive noise. Typical impulsive noise time period is from a few microseconds to milliseconds. Within this paper,the Middelston's additive white class A noise (AWCN) model is used as a statistical model for the impulse noise [8]. This model provides both the colored and impulse noise. Where the received sample $r_{k}$ at time instant $k$ is represented by

$$
\begin{gathered}
r_{k}=s_{k}+w_{k}+\xi_{k} \\
r_{k}=s_{k}+i_{k}
\end{gathered}
$$

Where $S_{k}$ is the transmitted sample, $\xi_{k}$ and $\mathbf{W}_{\mathrm{k}}$ are the complex impulse and Gaussian colored noise samples, and $i_{k}$ stands for the complex channel noise sample that collects the impulse noise and colored noise samples. A two states impulse noise model can be applied for modelling AWCN channel where the colored and impulse noise is picked up with the probability distribution function $i_{k}$ is given by

$p\left(i_{k}\right)=(1-c) \frac{1}{2 \pi \sigma_{0}{ }^{2}} e^{\left(-\frac{\left|i_{k}\right|^{2}}{2 \sigma_{0}{ }^{2}}\right)}+c \frac{1}{2 \pi \sigma_{1}^{2}} e^{\left(-\frac{\left|i_{k}\right|^{2}}{2 \sigma_{1}^{2}}\right)}$,

$\sigma_{0}{ }^{2}=\sigma_{w}{ }^{2}, \sigma_{1}{ }^{2}=\frac{\sigma_{\xi}^{2}}{A}+\sigma_{w}{ }^{2}$

Where $\mathrm{c}$ is the impulse noise index and is given by the multiplication of the average count of impulses per unit time and the average duration of the emmitted impulses enering the receiver [9]-[11]. In other words $c$ is the probability of appearance of impulsive noise, $a$ is the lowest value of interest and $b$ is the highest value of interest. The probability density function (PDF) of impulsive noise is written as

$$
f(x)=\frac{c}{b-a}+(1-c) \delta(x)
$$

In this paper in order to test the propoesed SC-FDE system over different types of impulsive noise distribution and conditions, the following parameters are set to be as: $a=-10, b=10$ and three types of impulsive noise levels (weak, medium and high) $\mathrm{c}=0.001, \mathrm{c}=0.003$ and $\mathrm{c}=0.006$ respectively. It is worth to mention here that 


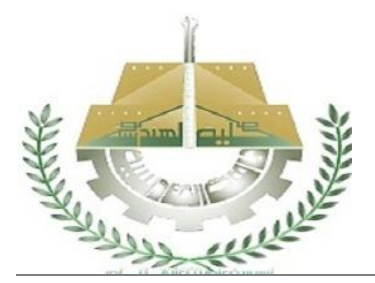

JOURNAL OF AL-QADISIYAH FOR ENGINEERING SCIENCE

\section{Vol.11, No. 2}

ISSN: $1998-4456$

the system performance will be changed as these values of $c$ changed, however, the chosen values are the most practical and real case.

As the impulsive noise power is much more eminent than the average power of the thermal noise, the use of FFT in multicarrier modulation will average this high noise power among the all subcarriers spectrums which lead to whole system performance degradation. Consequently, a SC-FDE system is purported in this paper with effective interleaving technique to mitigate the ramifications effect of such interference on the system operation.

\section{SC-FDE SYSTEM WITH THE IMPULSIVE NOISE CHANNEL}

Consider the SC-FDE system mode [12], which is shown in Figure 1. The input data are first mapped into a specific constellation and the ith time domain block of $\mathrm{N}$-points incoming modulated data vector, which is chosen uniformly from QAM or PSK constellations. Finally, the input data is passing through a mapper to produce a digitally encoded symbols. In the paperwork, we will use BPSK phase shift keying and QAM. These mapped symbols are found in vectors, each of $N$ symbols. It follows that, a cyclic prefix of length $M=N / 4$ is added to each transmit symbol to make the vector This signal is then given through the PLC channel with multipath effect, additive white Gaussian noise (AWGN) and driving noise $w_{k}$ (AWGN) and impulsive noise $\left(\xi_{n}\right)$. The received signal is then written as $x_{n}$ where $n=0,1, \ldots \ldots(N+M-1)$

$$
y_{n}=x_{n} * g_{n}+w_{n}+\xi_{n}
$$

Where, $g_{n}$ is the channel impulse response, $w_{n}$ is the AWGN and $\xi_{n}$ is the impulsive noise.

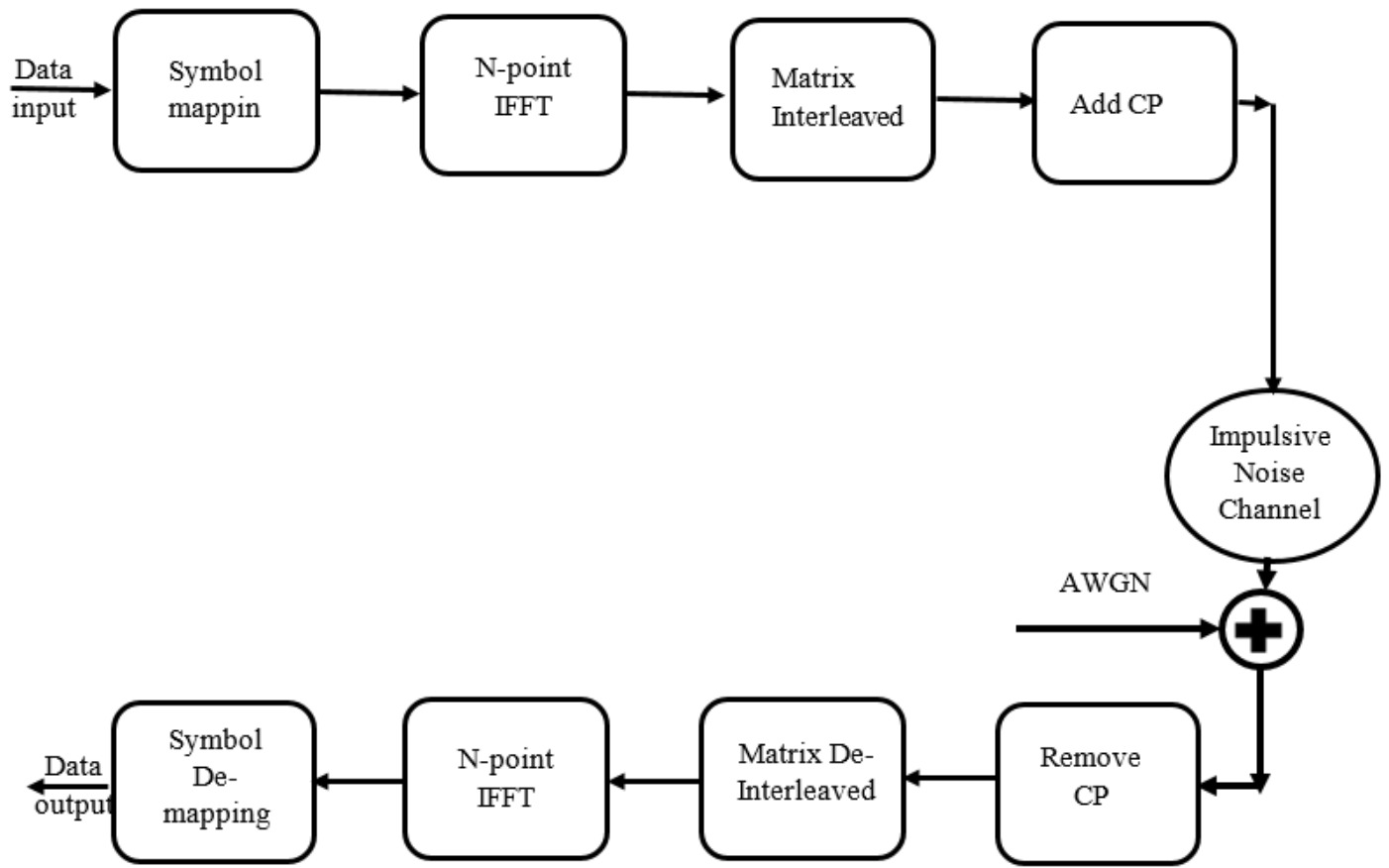

Figure 1. Standard matrix-interleaved Multicarrier (MI-MC) system block diagram. 

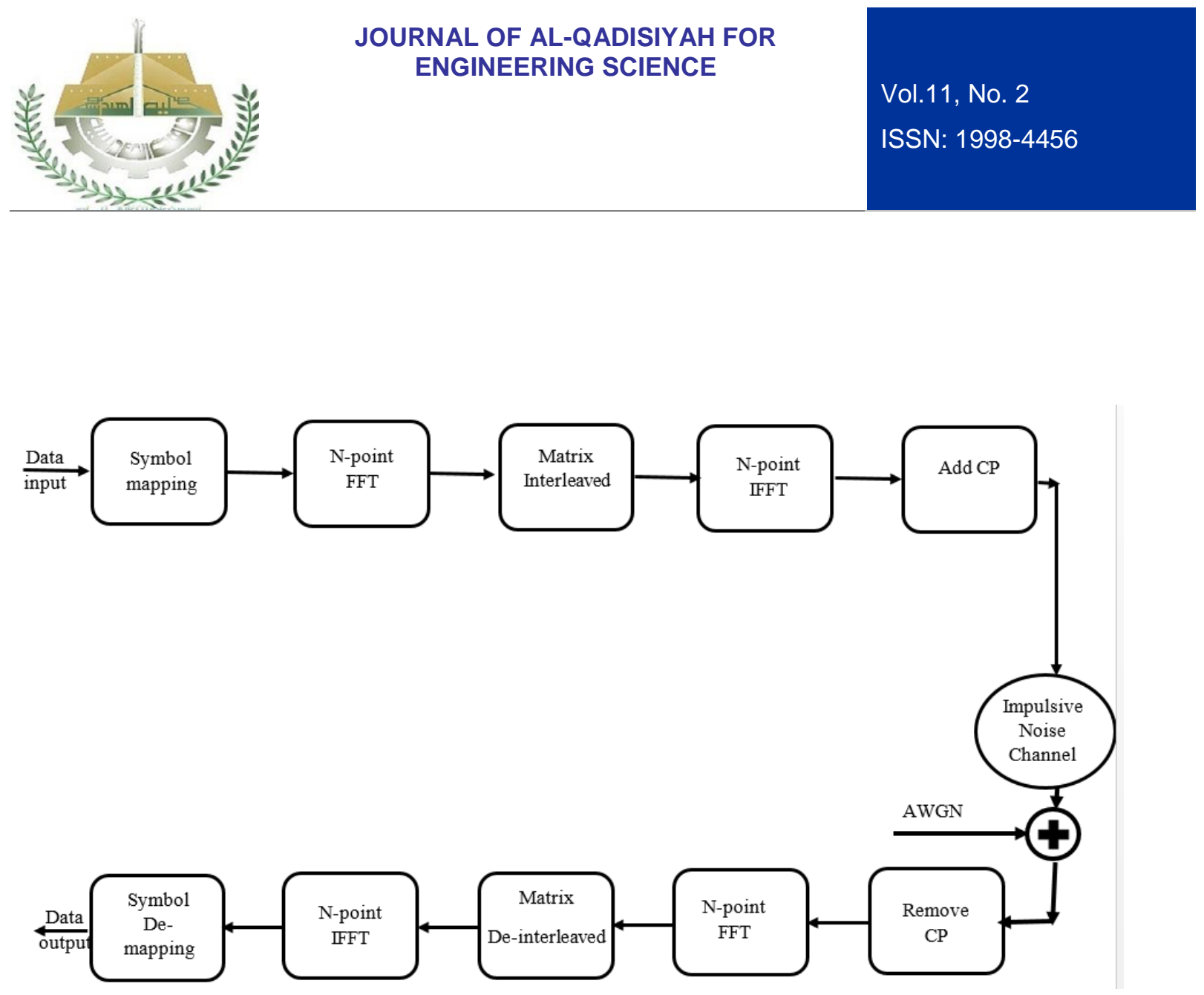

Figure 2. Proposed Matrix-Interleaved SC-FDE system block diagram.

On the receiver side, the cyclic prefix is first removed to cancel the effect of inter-symbol interference (ISI). Under the assumption of perfect frequency

$$
r=F Y
$$

Where $F$ is the discrete Fourier transform matrix and

$$
Y=\left[\begin{array}{llllll}
y_{1} & y_{2} & y_{3} & \ldots \ldots & \ldots & y_{n-1}
\end{array}\right]^{T}
$$

Thus,

$$
r_{n}=S_{n} \cdot G_{n}+w_{n} \cdot \xi_{n}
$$

Where $S_{n}=\sum_{m=1}^{N-1} y_{m} e^{\frac{-j 2 \pi m n}{N}}$, is the Fourier transform of $y_{n}, G_{n}$ is channel transfer function, $w_{n}$ and $\xi_{n}$ is Fourier transform of AWGN and impulsive noise respectively.

Frequency domain zero forcing single tap equalizer is then performed to remove the effect of the channel

$$
r_{n}^{Z F}=S_{n}+\frac{w_{n}}{G_{n}}+\frac{\xi_{n}}{G_{n}}
$$

Inverse DFT is then applied on (8) to produce

$$
\hat{x}_{n}=x_{n}+\sum_{m=0}^{N} \frac{w_{m}}{G_{m}} e^{\frac{j 2 \pi m n}{N}}+\sum_{m=0}^{N} \frac{\xi_{n}}{G_{n}} e^{\frac{j 2 \pi m n}{N}}
$$




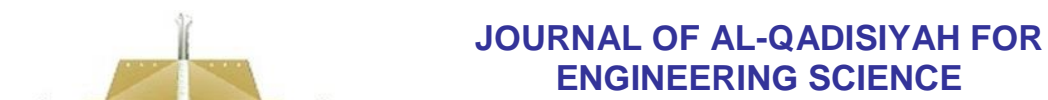

Vol.11, No. 2

ISSN: $1998-4456$

\subsection{PERFORMANCE ANALYSIS OF SC-FDE}

The total error signal is the difference between transmitted and receive signal $e=\left(x_{n}-\hat{x}_{n}\right)$ then, $e=$ $\sum_{m=0}^{N} \frac{w_{m}}{G_{m}} e^{\frac{j 2 \pi m n}{N}}+\sum_{m=0}^{N} \frac{\xi_{n}}{G_{n}} e^{\frac{j 2 \pi m n}{N}}$

The signal -to-noise ratio (SNR) is

$$
\begin{gathered}
S N R=\frac{\text { Signal power }}{\text { Noise power }}=\frac{E\left[x_{n} \cdot x_{n}^{*}\right]}{E\left[e \cdot e^{*}\right]} \\
=\frac{\delta_{S}^{2}}{\delta_{n}^{2} \sum_{m=0}^{N-1} \frac{1}{\left|G_{m}\right|^{2}}+\delta_{\zeta}^{2} \sum_{m=0}^{N-1} \frac{1}{\left|G_{m}\right|^{2}}} \\
=\frac{\delta_{S}^{2}}{\left(\delta_{n}^{2}+\delta_{\zeta}^{2}\right) \sum_{m=0}^{N-1} \frac{1}{\left|G_{m}\right|^{2}}}
\end{gathered}
$$

Where $\delta_{S}^{2}$ is the signal power, $\delta_{n}^{2}$ is the standard deviation of the AWGN (AWGN noise power), $\delta_{\zeta}^{2}$ is the impulsive noise power.

\subsection{NUMERICAL RESULTS}

To investigate the old and proposed systems under the AWGN and impulsive noise, Matlab (Monte Carlo) simulation is used to clear up the superiority of the time domain interleaved single carrier frequency domain equalizer (SC-FDE) over the interleaved multicarrier system. Number of subcarriers used in this simulation is 1024 with a 256 subcarriers are used as a guard interval to prevent the intersymbol interference (ISI). The simulation is run for different impulsive noise level scenarios to certain the validity of our proposed scheme over the conventional system.

The BER performance of the proposed SC-FDE is introduced and compared with the conventional MC system over impulsive noise channel is shown in Figures 1 and 2 for the QPSK and 16-QAM modulation formats respectively. For fair comparison, time domain matrix interleaved is used in both proposed scheme and the classic MC system. It is clear from Figure 1 that, for QPSK data mapping, the proposed matrix interleaved MISC-FDE is superior the standard MI-MC system for different levels of probability of appearance of impulsive noise $\mathrm{c}=0.001, \mathrm{C}=0.003$ and $\mathrm{c}=0.006$. We take these three values of $\mathrm{c}$ (weak, medium and high) impulsive noise to validate the proposed scheme performance under different types of operating conditions. It is also clear that unlike the proposed MI-SC-FDE system, the classic MI-MC system suffers from error floors for medium and high impulsive noise levels $\mathrm{c}=0.003$ and $\mathrm{c}=0.006$ at low signal-to-noise ratio ( $\mathrm{SNR}=8 \mathrm{~dB}$ ) whereas our proposed scheme BER still decreases rapidly as SNR increases without any error floors for even medium and high impulsive noise levels.

To further investigate the superiority of the proposed MI-SC-FDE scheme over the standard MI-MC system, 16-QAM data mapping is also taken into consideration and shown in Figure 2. Figure 2 shows the BER performance of the proposed MI-SC-FDE and compared it with the BER performance of the standard MI-MC system for different levels of impulsive noise and 16-QAM constellation. One can notice that the proposed scheme still better BER performance than the classic scheme. The classic MI-MC scheme still suffers from error floors at $\mathrm{C}=0.003$ and $\mathrm{c}=0.006$ at $\mathrm{SNR}=13 \mathrm{~dB}$. However, the proposed MI-SC-FDE scheme still robust to impulsive noise and error floors even for high SNR and high impulsive noise levels. The superiority of the proposed scheme becomes more significant as the severity of impulsive noise increased. 

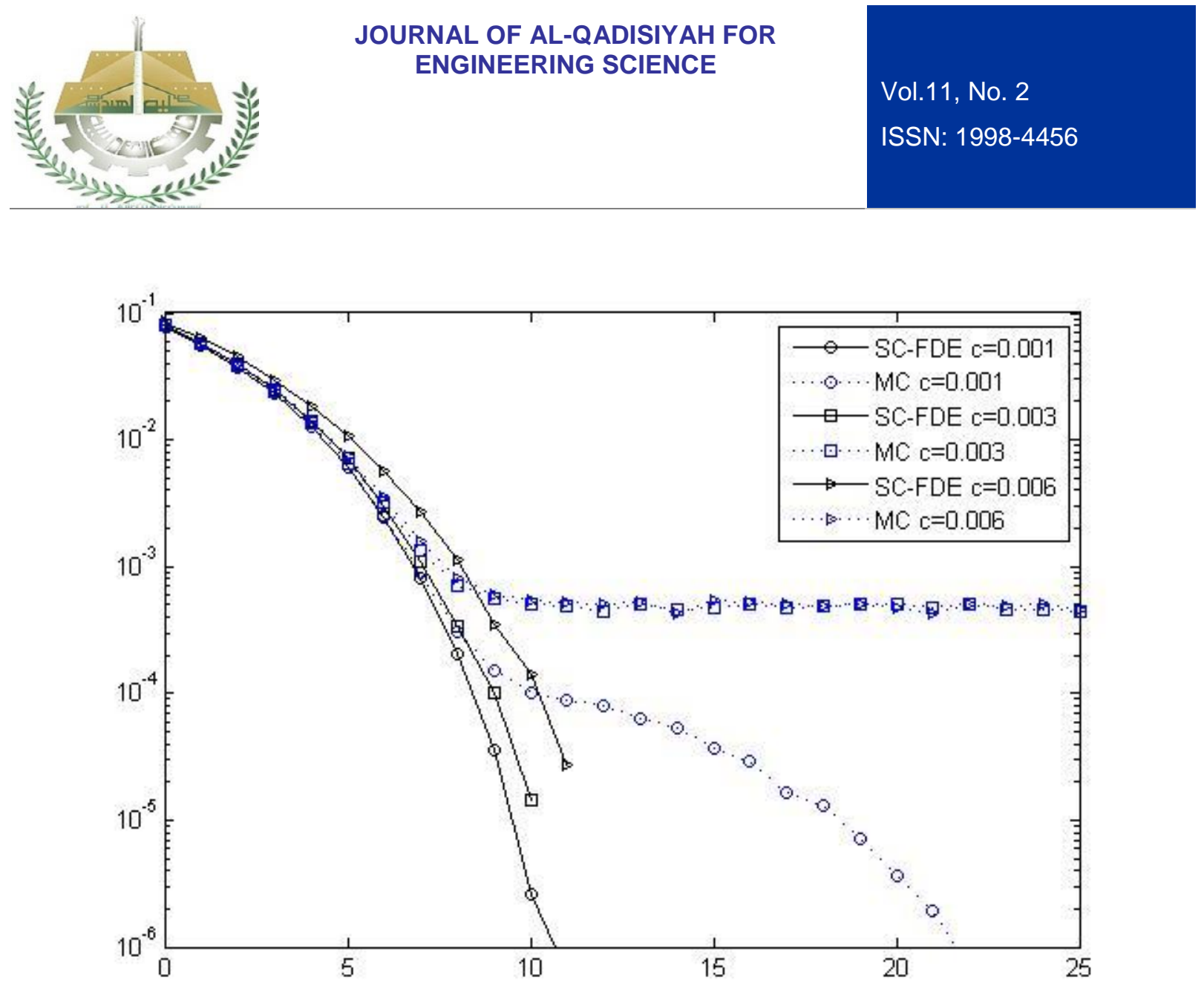

Figure 3. BER performance of the proposed MI-SC-FDE and the standard MI-MC systems in the case of QPSK data mapping and over impulsive noise channel. 

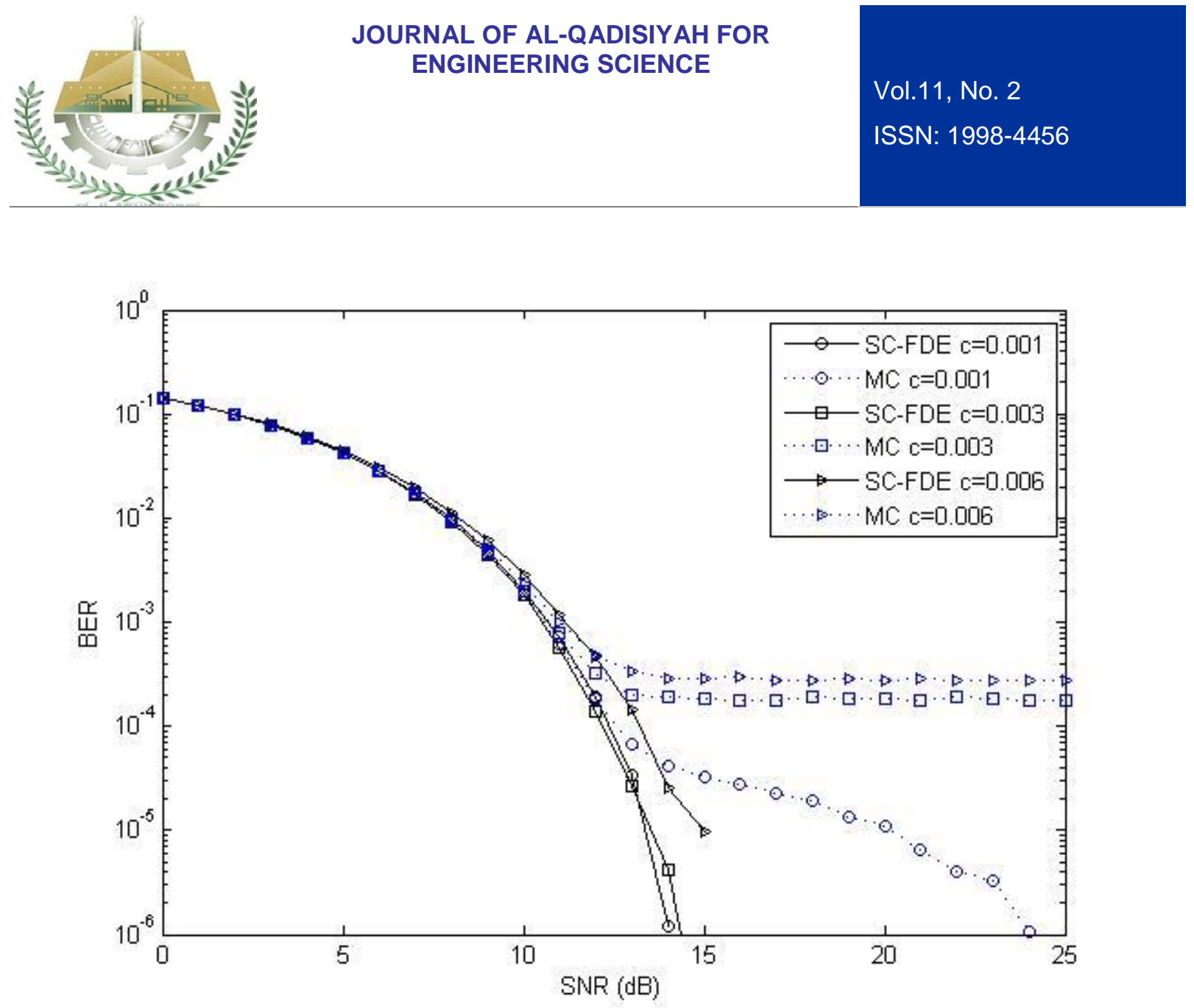

Figure 4. BER performance of the proposed MI-SC-FDE and the standard MI-MC systems in the case of 16QAM data mapping and over impulsive noise channel.

\subsection{CONCLUSIONS}

A new time matrix interleaved SC-FDE system is presented to cope with the severity of impulsive noise channels. Simulation results proved the superiority of the proposed MI-SC-FDE over the standard MImulticarrier system for different impulsive noise levels and data mapping. Unlike the conventional MC system, the BER of the proposed system continues to decrease rapidly as the SNR increases and does not suffer from any error floors. However, the conventional MI-MC system sufferes from error floors at low level of SNR in the case of medium and high impulsive noise. The proposed MI-SC-FDE has proved itself as an efficient technique to eliminate the deleterious effects of impulsive noise and achieved good BER performance in comparison to the standard MI-multicarrier system.

\section{REFERENCES}

1. Fabricio, P.; Giorgio,M.V.; Reza, K.; Naofal. A; Morat, U.; Hakam, M.Single., Carrier Frequency Domain Equalization," IEEE signal Processing Magazine". Sept. 2008.

2. Thokozani, S.; Han Vinck, A.J; Hendrik, C. F., A Study on Implusive noise and its models," The 4th World Conference on Educational Sciences", WCES-2012, Barcelona, Spain, 2-5 february 2012, pg. 128-132.

3. F. Rouissi, H. Gassara, A. Ghazel and S. Najjar, Comparative Study of Impulse Noise Models in the Narrow Band Indoor PLC Environment," tenthe workshop on power line communications", , 10-11 OCTOBER, 2016, Paris, France. 


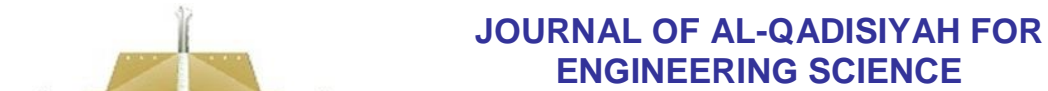

ENGINEERING SCIENCE
Vol.11, No. 2

ISSN: $1998-4456$

4. Kapil Gulati, Aditya Chopra, Brian L. Evans and Keith R. Tinsley., Statistical Modeling of Co-Channel Interference," Acoustics Speech and Signal Processing (ICASSP)" 2010 IEEE International Conference on 14-19 March 2010 Dallas, TX, USA.

5. Marcel Nassar, Kapil Gulati, Yousof Mortazavi and Brian L. Evans., Statistical Modeling of Asynchronous Impulsive Noise in Powerline Communication Networks," Global Telecommunications Conference (GLOBECOM 2011)" 2011 IEEE, 5-9 Dec. 2011, Kathmandu, Nepal.

6. Namrata Jain, Namrata Sahayam, Iterative Looping for Impulsive Noise Channels in QAM-OFDM Based Systems" International Journal of Emerging Technology and Advanced Engineering" Vol. 3, Issue 11, November 2013 .

7. Amit Grover and ${ }^{* *}$ Amit Kumar Kohli, Simulation pf implusive noise environment for othogonal frequency division multiplexing wireless systems," International Journal of Computing \& Business Research" ISSN (Online): 2229-6166.

8. Vinay Chakravarthi Gogineni1, Subrahmanyam Mula., Proportionate Adaptive Filtering under Correntropy Criterion in Impulsive Noise Environments," arXiv:1707.00315v1 [cs.SY] 2 Jul 2017.

9. Alexey Ruchay and Vitaly Kober., Impulsive noise removal from color images with morphological filtering," The 6th international conference on analysis of images, social networks, and texts" (AIST 2017), 27-29 July, 2017, Moscow, Russia.

10. Jihad Fahs, Ibrahim Abou-Faycal., Information Measures, Inequalities and Performance Bounds for Parameter Estimation in Impulsive Noise Environments." arXiv:1609.00832 .

11. Marcel Nassar, Philip Schniter, and Brian L. Evans., A Factor Graph Approach to Joint OFDM Channel Estimation and Decoding in Impulsive Noise Environments," IEEE Transactions on Signal Processing," arXiv:1306.1851.

12. A. Al-Dweik1 3, A. Hazmi2, B. Sharif3 and C. Tsimenidis3 , Effecient interleaving Techniques for OFDM System over Impulsive Noise Channels," 2010 IEEE 21st International Symposium on Personal Indoor and Mobile Radio Communications. 